\title{
Evolution of nutrient resorption across the herbaceous genus Helianthus
}

\author{
Ashley M. Rea 1 Chase. M. Mason • Lisa A. Donovan
}

Received: 2 November 2017/Accepted: 16 May 2018/Published online: 28 May 2018

(C) The Author(s) 2018

\begin{abstract}
Foliar nutrient resorption is a key modulator of plant nutrient use. However, evolutionary patterns for nutrient resorption remain unclear, especially in herbs. We measured nitrogen and phosphorus resorption on preselected leaves across the Helianthus (sunflower) genus in a common garden in Athens, GA. We analyzed our data with published leaf traits and native habitat environmental data. Using phylogenetically controlled analyses, we tested if (1) nutrient resorption correlates with leaf economic, vasculature, and defense traits through evolutionary time, and (2) native habitat environment predicts nutrient resorption evolution. For Helianthus, nutrient resorption capacity is greater in resource-conservative species, as previously defined for Helianthus with a principle components analysis of leaf economic spectrum traits (photosynthetic rate, respiration rate, leaf lifespan, leaf mass per area, and green-leaf $\mathrm{N}$ and $\mathrm{P}$ concentrations). Nutrient resorption capacity also evolutionarily correlates with individual leaf economic traits, though not always as expected based on broad species
\end{abstract}

Communicated by Wayne Polley.

A. M. Rea ( $\varangle)$ · Chase. M. Mason · L. A. Donovan Department of Plant Biology, University of Georgia, Athens, GA 30602, USA

e-mail: ashleyreata@gmail.com

Chase. M. Mason

Department of Biology, University of Central Florida, Orlando, FL 32816, USA surveys. Greater nutrient resorption is also positively associated with leaf chemical defenses, but not leaf vasculature or senescence rate. Finally, nitrogen resorption evolution increases with native habitat precipitation, but native habitat soil fertility does not predict nitrogen or phosphorus resorption. Our results suggest nutrient resorption evolution is more closely tied to resource economic strategy than native habitat.

Keywords Nutrient resorption · Traits · Leaf economics spectrum · Tannins · Selection $\cdot$ Sunflowers

\section{Introduction}

Nutrient resorption is fundamental to plant nutrient economy. Resorption lowers net leaf nutrient construction costs, which somewhat frees plants from strict dependence on nutrient uptake capacity or availability (Aerts 1996; Chapin 1989; Eckstein et al. 1999; Zhang et al. 2015). Nutrient resorption may also contribute to an over-arching resource use strategy, which may result in predictive relationships between nutrient resorption and other traits related to how plants acquire, conserve, and defend nutrients in the varying climatic and soil environments plants contend with as they evolve.

One set of resource-use strategies is the leaf economics spectrum (LES). The LES is a pattern of 
leaf-level tradeoffs between traits conveying productivity and traits conveying persistence (Diaz et al. 2004; Reich 2014; Wright et al. 2004). High-productivity traits are found in "resource-acquisitive" species, and include high photosynthetic and respiration rates, high leaf nitrogen $(\mathrm{N})$ and phosphorus (P) concentration, and low leaf mass per area (LMA) and leaf lifespan. Species toward the resource-acquisitive end of this spectrum rapidly repay initial carbon investment of leaf production at the expense of thinner, flimsier leaves that senesce sooner. Resource-conservative species generally have longlived, durable leaves, but the structural carbon required for persistence results in lower productivity per unit time (Diaz et al. 2004; Reich 2014; Wright et al. 2004).

We hypothesize that resource-conservative species resorb more foliar nutrients than resource-acquisitive species. High nutrient resorption capacity may allow resource-conservative species to thrive despite their low nutritional status by increasing nutrient mean retention time (Wright and Westoby 2003; Zhang et al. 2015). Furthermore, resource-conservative species have longer-lived leaves, which may contribute to slower leaf senescence rates that allow more time for resorption. Studies examining nutrient resorption and the LES have mixed results, with some suggesting higher nutrient resorption is a resource-conservative trait (Wood et al. 2011; Wright and Westoby 2003; Zhang et al. 2015) and others concluding resorption is independent of resource use strategy (Freschet et al. 2010).

Leaf economic theory predicts nutrient-conservative, longer-lived leaves are also better protected (Diaz et al. 2004; Mason et al. 2016; Mason and Donovan 2015b). If higher resorption capacity is a resourceconservative trait, leaf defense compounds such as phenolics and tannins should increase along with resorption to protect hard-won nutrients from loss through herbivory. Mason et al. (2016) found tannin concentrations are higher in more resource-conservative Helianthus species. However, Aerts (1997) hypothesized phenolics precipitate leaf proteins during senescence and make them unavailable for resorption. If phenolics physiologically impede nutrient resorption, then evolution of high chemical defense via tannins may preclude evolution of high nutrient resorption and generate a negative relationship between these traits among taxa.
Nutrient resorption capacity may also have coevolved with leaf vascular capacity due to mechanistic physiological dependencies. Leaf vein density, a common proxy for vascular capacity, plays an important role in nutrient metabolism and transport (Sack et al. 2012, 2013; Zhang et al. 2015). If evolution of high resorption capacity requires high vascular capacity for export from senescing leaves, vein density and nutrient resorption may have a positive relationship. Based on these interactions leaf chemical defenses, and vascular capacity as described by leaf vein density may all be evolutionarily correlated with resorption in Helianthus.

Whether the result of physiological dependencies or trade-offs, all trait-trait correlations evolve in the context of native habitat. Large-scale studies show nutrient resorption generally decreasing as habitat fertility or green-leaf nutrient concentration increases (Aerts 1996; Killingbeck 1996; Kobe et al. 2005; Wright and Westoby 2003; Yuan and Chen 2009). However, it is unclear if this trend is evolutionary or a plastic phenotypic response to soil fertility (Aerts 1996). Though many researchers have hypothesized increased nutrient resorption explains plant adaptation to infertile environments (Eckstein et al. 1999; Zhang et al. 2015; Distel et al. 2003), this has not always been supported by empirical evidence (Chapin 1980; Aerts 1996; Aerts and Chapin 2000; Brant and Chen 2015; Drenovsky and Richards 2006). If nutrient resorption enables plant survival in low-fertility conditions, species from lower-fertility habits should express higher capacity for nutrient resorption than species from higher-fertility habitats.

Nutrient resorption capacity is measured as either resorption efficiency or resorption proficiency. Resorption efficiency is the proportion of resorbed nutrients relative to green-leaf nutrients. Resorptionproficiency measures terminal resorption ability of the leaf and represents the biochemical limit of resorption; more proficient leaves have lower senesced-leaf nutrient levels (Killingbeck 1996). Although efficiency and proficiency are complementary traits, Killingbeck (1996) argues proficiency is under stronger selection and more useful in evolutionary studies. In contrast, efficiency is useful for comparing species' relative capabilities of retaining acquired nutrients (Killingbeck 1996).

We used the Helianthus (sunflower) genus to explore the connection between nutrient resorption 
evolution and other leaf traits and native habitat. Helianthus includes a diverse mix of annual and perennial species and spans a range of North American habitats with varying climate and soil characteristics (Heiser et al. 1969; Mason and Donovan 2015b; Sack et al. 2013). This is a good system for examining nutrient resorption because Helianthus species have been shown to vary in leaf economic traits and associated resource-use strategies (Mason and Donovan 2015b), defense traits (Mason et al. 2016; Mason and Donovan 2015a), vascular traits (Mason and Donovan 2015b), and secondary metabolites (Webber and Mason 2016), as well as stem and root traits (Bowsher et al. 2016; Pilote and Donovan 2016). Our study captured patterns of trait expression by minimizing environmentally induced plasticity using a common garden (de Villemereuil et al. 2016; Donovan et al. 2014). We examined nutrient resorption variation through evolutionary time in the light of physiological trade-offs and resource availability in native habitats. We used novel nutrient resorption data and published leaf and habitat environment data for 28 sunflower species of known phylogenetic relationship (Stephens et al. 2015). Specifically, we asked (1) if nutrient resorption correlates through evolutionary time with LES, leaf vascular, and leaf defense traits, and (2) if native habitat environment predicts nutrient resorption evolution.

\section{Methods}

Plant growth

We present previously unpublished nutrient resorption data from a published common garden experiment assessing evolution of leaf economic and defense traits (Mason et al. 2016; Mason and Donovan 2015b; Webber and Mason 2016) (Table 1). Seeds from two to four populations each of 27 Helianthus annual and perennial species and one Phoebanthus species were either wild collected or obtained from accessions maintained by the USDA National Genetic Resources Program. These diploid, nonhybrid species are evenly distributed across the most recent Helianthus phylogeny (Stephens et al. 2015). Populations are spread across each species range (83 populations total). For more information on population selection and seed sourcing, see (Mason and Donovan 2015b).

Eight replications of all populations were grown under high-resource conditions in identical pots and soil mixtures. The same experiment has additionally been used to assess floral trait evolution (Mason et al. 2017b), biomass allocation, and reproductive timing (Mason et al. 2017a). A detailed account of experimental conditions can be found in Mason and Donovan (2015b). See Table 1 for sources of all published data used in this study.

Leaf selection and trait measurement

To avoid confounding effects of leaf ontogeny on nutrient resorption and other leaf trait values (Mason et al. 2013; van Heerwaarden et al. 2003), leaves were predesignated for collection. The most recent fully expanded leaf (MRFEL) and its oppositely paired leaf were collected between the fourth leaf pair stage and the onset of reproduction and the newly expanding leaf pair immediately above was tagged. The tagged leaf pair was monitored for loss-of-greenness three times per week and harvested directly off the plant at full senescence (Mason and Donovan 2015b). We calculated leaf senescence rate as the number of days between $25 \%$ loss-of-greenness and $100 \%$ loss-ofgreenness. Four undamaged senesced leaves from different replicate plants within each population were sampled for $\mathrm{N}$ and $\mathrm{P}$ concentrations for this study.
Table 1 Literature base on which the dataset was compiled for analysis with $\mathrm{N}$ - and P-resorption efficiencies and proficiencies

\begin{tabular}{ll}
\hline Trait(s) & Source \\
\hline LES PC1 & Mason and Donovan (2015b) \\
Leaf lifespan, LMA, photosynthetic rate, respiration rate & Mason and Donovan (2015b) \\
Green-leaf N, P, N:P, and C:N & Mason and Donovan (2015b) \\
Tannin activity & Mason et al. (2016) \\
Flavonoids and total phenolics & Webber and Mason (2016) \\
Native habitat environmental traits & Mason and Donovan (2015b) \\
\hline
\end{tabular}


All leaf economic traits, including green-leaf $\mathrm{N}$ and $P$ concentrations, were measured on the MRFEL (Mason and Donovan 2015b). Green-leaf tannin activity (as determined by protein-precipitation capacity), total phenolics, flavonoids, and other chemical and structural leaf defenses were measured on the MRFEL (Mason et al. 2016; Webber and Mason 2016).Vein density was measured on the oppositely paired leaf (Mason and Donovan 2015b).

Leaf $\mathrm{N}$ and $\mathrm{P}$ concentrations were measured in leaves which were dried in a forced-air drying oven at $60{ }^{\circ} \mathrm{C}$ for $72 \mathrm{~h}$ before being finely ground with a ball mill. $\mathrm{N}$ concentration was determined via carbonnitrogen analysis using Micro-Dumas Combustion (NA1500, Carlo Erba Strumentazione, Milan, Italy) at the University of Georgia Analytical Chemistry Laboratory. P concentration was determined via acidmolybdate spectrophotometry on ashed leaf powder (Varvel et al. 1976).

We calculated resorption efficiency sensu Killingbeck (1996) as:

Resorption efficiency $(\%)=\frac{\text { nutrients }_{\mathrm{GL}}-\text { nutrients }_{\mathrm{SL}}}{\text { nutrients }_{\mathrm{GL}}}$

where nutrients $\mathrm{GL}_{\mathrm{GL}}$ is the population average green-leaf nutrient concentration and nutrients $\mathrm{SL}_{\mathrm{SL}}$ is the population average senesced-leaf nutrient concentration. We calculated resorption proficiency sensu Killingbeck (1996) as:

Resorption proficiency $=$ nutrients $_{\mathrm{SL}}$

\section{Environmental data}

Source habitat soils and climate data were obtained for all 83 populations as described in Mason and Donovan (2015b). Five soil cores were collected from each population source habitat, dried at $60{ }^{\circ} \mathrm{C}$ in a forcedair drying oven for $72 \mathrm{~h}$, and homogenized. Each core was analyzed for $\mathrm{pH}$, cation exchange capacity, organic matter content, and soil chemistry (phosphorus, potassium, magnesium, calcium) by A\&L Eastern Laboratories (Richmond VA). N, carbon (C), and C:N ratio was determined using Micro-Dumas Combustion at the University of Georgia Analytical Chemistry Laboratory. Replicate cores were averaged; these source habitat means were used for all subsequent analysis.

Temperature and precipitation data were obtained for each population from the WorldClim database (Hijmans et al. 2005). Aridity Index and potential evapotranspiration (PET) data were obtained from the CGIAR Global Aridity and PET database (Zomer et al. 2008).

Statistical analysis

Recent critiques in LES literature have addressed normalizations in trait calculation. Mathematical interconversion of mass- and area-based measurements using LMA causes spurious correlations because LMA varies more than traits like nutrient concentration (Lloyd et al. 2013; Osnas et al. 2013; Pearson 1897; Poorter et al. 2014; Tu 2016). In this study, we performed normalizations when we calculated area-based resorption proficiency by dividing senesced-leaf nutrient concentration by green-leaf LMA. However, critiques of nutrient resorption measurement techniques argue area-based normalizations are more reliable because mass-based senesced-leaf $\mathrm{N}$ and $\mathrm{P}$ may be biased by carbon resorption. Carbon resorption impacts the senesced-leaf's overall mass and therefore the concentration of the remaining nutrients in the senesced-leaf (Kandil et al. 2004; van Heerwaarden et al. 2003). To address these possibilities, we present and discuss our results using mass- and area-based normalizations (Poorter et al. 2014; Westoby et al. 2013).

In comparative analysis among species, phylogenetic signal can confound results because it can lead to trait correlation due to shared ancestry rather than adaptation or biophysical constraints (Felsenstein 1985). We tested for phylogenetic signal with Blomberg's $\mathrm{K}$ and Pagel's $\lambda$ using the phylosig function in the phytools $\mathrm{R}$ package (Blomberg et al. 2003; Pagel 1999; Revell 2012). While no resorption traits displayed significant phylogenetic signal, many of the previously published leaf traits do, therefore we used phylogenetically explicit analyses.

Evolutionary trait-trait and trait-environment correlations were assessed on population-weighted species means with the phylopars function in the $\mathrm{R}$ package Rphylopars (Paradis et al. 2004; Goolsby 2016; Martins and Garland 1991). To assess correlations between nutrient resorption and the LES, we 
Table $2 \mathrm{r}^{2}$ of evolutionary trait-trait correlations on population-weighted species means for (a) mass- and (b) area-normalized data

\begin{tabular}{|c|c|c|c|c|c|c|c|c|c|}
\hline 2 (a) & $\mathrm{P}_{\mathrm{eff}}$ & $\mathrm{P}_{\mathrm{SL}}$ & $\mathrm{N}_{\text {eff }}$ & $\mathrm{N}_{\mathrm{SL}}$ & $\mathrm{N}_{\text {eff }}: \mathrm{P}_{\text {eff }}$ & $\mathrm{N}_{\mathrm{SL}}: \mathrm{P}_{\mathrm{SL}}$ & Flavonoid & Phenol & LSR \\
\hline$P_{\text {eff }}$ & 1 & & & & & & & & \\
\hline $\mathrm{P}_{\mathrm{SL}}$ & (-) 0.57 & 1 & & & & & & & \\
\hline $\mathrm{N}_{\mathrm{eff}}$ & (+) 0.35 & (-) 0.38 & 1 & & & & & & \\
\hline $\mathrm{N}_{\mathrm{SL}}$ & (-) 0.37 & $(+) 0.48$ & (-) 0.82 & 1 & & & & & \\
\hline $\mathrm{N}_{\mathrm{eff}}: \mathrm{P}_{\mathrm{eff}}$ & $(-) 0.63$ & $(+) 0.37$ & - & - & 1 & & & & \\
\hline $\mathrm{N}_{\mathrm{SL}}: \mathrm{P}_{\mathrm{SL}}$ & - & - & - & - & - & 1 & & & \\
\hline Flavonoid & - & - & - & - & - & - & 1 & & \\
\hline Phenol & - & (-) 0.21 & (+) 0.21 & $(-) 0.27$ & - & - & (+) 0.99 & 1 & \\
\hline LSR & - & - & - & - & - & - & - & - & 1 \\
\hline $\mathrm{A}_{\text {mass }}$ & - & - & - & - & - & - & - & - & - \\
\hline $\mathrm{R}_{\text {mass }}$ & (-) 0.25 & (+) 0.39 & - & - & (+) 0.44 & - & - & (-) 0.17 & - \\
\hline LMA & - & - & - & - & $\mathrm{v}$ & - & - & - & - \\
\hline LL & - & - & - & $(+) 0.15$ & - & - & - & - & (+) 0.19 \\
\hline $\mathrm{N}_{\mathrm{GL}}$ & - & - & - & - & - & - & - & - & - \\
\hline $\mathrm{P}_{\mathrm{GL}}$ & - & - & - & - & - & - & - & - & - \\
\hline $\mathrm{N}_{\mathrm{GL}}: \mathrm{P}_{\mathrm{GL}}$ & - & - & - & - & - & - & - & - & - \\
\hline $\mathrm{C}_{\mathrm{GL}}: \mathrm{N}_{\mathrm{GL}}$ & - & - & - & $\mathrm{v}$ & $\mathrm{v}$ & - & - & - & - \\
\hline Tannin & - & - & - & $(-) 0.18$ & - & - & - & - & - \\
\hline VD & - & - & - & - & - & - & - & - & - \\
\hline LESPC1 & - & - & - & - & - & - & - & - & - \\
\hline 2 (b) & $P_{\text {eff }}$ & $\mathrm{P}_{\mathrm{SL}}$ & $\mathrm{N}_{\text {eff }}$ & $\mathrm{N}_{\mathrm{SL}}$ & $\mathrm{N}_{\mathrm{eff}}: \mathrm{P}_{\mathrm{eff}}$ & $\mathrm{N}_{\mathrm{SL}}: \mathrm{P}_{\mathrm{SL}}$ & Flavonoid & Phenol & LSR \\
\hline$P_{\text {eff }}$ & 1 & & & & & & & & \\
\hline $\mathrm{P}_{\mathrm{SL}}$ & $(-) 0.42$ & 1 & & & & & & & \\
\hline $\mathrm{N}_{\mathrm{eff}}$ & 0.35 & (-) 0.41 & 1 & & & & & & \\
\hline $\mathrm{N}_{\mathrm{SL}}$ & (-) 0.15 & (+) 0.48 & (-) 0.74 & 1 & & & & & \\
\hline $\mathrm{N}_{\mathrm{eff}}: \mathrm{P}_{\mathrm{eff}}$ & $(-) 0.63$ & $(+) 0.22$ & - & - & 1 & & & & \\
\hline $\mathrm{N}_{\mathrm{SL}}: \mathrm{P}_{\mathrm{SL}}$ & - & - & - & - & - & 1 & & & \\
\hline Flavonoid & - & - & - & - & - & - & 1 & & \\
\hline Phenol & - & $(-) 0.17$ & - & $(-) 0.16$ & - & - & (+) 0.99 & 1 & \\
\hline LSR & - & - & - & - & - & - & - & - & 1 \\
\hline$A_{\text {area }}$ & - & - & - & (+) 0.27 & - & - & - & - & - \\
\hline $\mathrm{R}_{\text {area }}$ & $(-) 0.17$ & (+) 0.45 & - & - & (+) 0.23 & - & - & - & - \\
\hline LMA & - & - & - & $(+) 0.22$ & - & - & - & - & - \\
\hline LL & - & - & - & - & - & - & - & - & (+) 0.19 \\
\hline $\mathrm{N}_{\mathrm{GL}}$ & - & - & - & $(+) 0.22$ & - & - & - & - & - \\
\hline $\mathrm{P}_{\mathrm{GL}}$ & - & $(+) 0.31$ & - & $(+) 0.20$ & - & - & - & - & - \\
\hline $\mathrm{N}_{\mathrm{GL}}: \mathrm{P}_{\mathrm{GL}}$ & - & - & - & - & - & - & - & - & - \\
\hline $\mathrm{C}_{\mathrm{GL}}: \mathrm{N}_{\mathrm{GL}}$ & - & - & - & - & - & - & - & - & - \\
\hline Tannin & - & - & - & - & - & - & - & - & - \\
\hline VD & - & - & - & - & - & - & - & $\mathrm{v}$ & - \\
\hline
\end{tabular}


Table 2 continued

\begin{tabular}{llllllllll}
\hline $\mathbf{2}(\mathbf{b})$ & $\mathrm{P}_{\mathrm{eff}}$ & $\mathrm{P}_{\mathrm{SL}}$ & $\mathrm{N}_{\mathrm{eff}}$ & $\mathrm{N}_{\mathrm{SL}}$ & $\mathrm{N}_{\mathrm{eff}}: \mathrm{P}_{\text {eff }}$ & $\mathrm{N}_{\mathrm{SL}}: \mathrm{P}_{\mathrm{SL}}$ & Flavonoid & Phenol & LSR \\
\hline LESPC1 & - & $(+) \mathbf{0 . 3 3}$ & - & $(+) \mathbf{0 . 3 3}$ & - & - & - & - & - \\
\hline
\end{tabular}

All values shown are significant at $\mathrm{p} \leq 0.05$. Bold correlations are significant under FDR multiple comparisons. $P$ phosphorus, $N$ nitrogen, $P_{\text {eff }}$ P-resorption efficiency, $P_{S L}$ senesced-leaf $\mathrm{P}$ concentration, $N_{\text {eff }} \mathrm{N}$-resorption efficiency, $N_{S L}$ senesced-leaf $\mathrm{N}$ concentration, Flavonoid green-leaf flavonoid content, Phenol green-leaf phenolic content, $L S R$ leaf senescence rate, $A$ green-leaf photosynthetic rate, $R$ green-leaf nighttime respiration rate, $L M A$ green-leaf leaf mass per area, $L L$ leaf lifespan, $N_{G L}$ green-leaf $\mathrm{N}$ concentration, $P_{G L}$ green-leaf L P concentration, Tannin green-leaf tannin activity, $V D$ green-leaf vein density, $L E S P C 1$ principle components 1 of leaf economics spectrum traits

used published principal components analysis of the six traits comprising the LES (photosynthetic rate, respiration rate, leaf lifespan, LMA, and green-leaf $\mathrm{N}$ and P concentrations) (Mason and Donovan 2015b). For correlations between nutrient resorption and leaf economic traits, we used these six traits individually. Missing values were handled using restricted maximum likelihood optimization of covariance parameters (Bruggerman et al. 2009; Goolsby 2016). Multiple comparisons correction was performed using False Discovery Rate (FDR) (Benjamini and Hochberg 1995). Ancestral state reconstructions were performed using restricted maximum likelihood on the most recent Helianthus phylogeny with the phylopars function (Paradis et al. 2004; Goolsby 2016; Stephens et al. 2015). To perform phylogenetic least squares ANOVA, we used the $g l s$ function in the nlme package (Martins and Garland 1991; Pinheiro et al. 2014). We calculated p-values using the aov function. To detect differences between groups, we used the TUKEY HSD function in the stats package. Due to phylogenetically biased missing data for erect perennials from our flavonoid and total phenolics data, phylogenetic least squares ANOVA could not be performed for leaf phenolic or flavonoid content.

\section{Results}

Variation in nutrient resorption across Helianthus

The 28 species vary widely in $\mathrm{N}$ - and P-resorption efficiencies and proficiencies. Resorption efficiency varies almost twofold for $\mathrm{P}$ (57-93\%) and less so for $\mathrm{N}$ (69.5-90\%). N- and P-resorption proficiencies varies more than efficiency for both nutrients, with massbased senesced-leaf $\mathrm{N}$ varying threefold (0.42-1.5\%), mass-based senesced-leaf $\mathrm{P}$ varying sevenfold
(0.03-0.21\%), area-based senesced-leaf $\mathrm{N}$ varying fourfold (18.3-70.7 $\left.\mathrm{g} / \mathrm{m}^{2}\right)$, and area-based senescedleaf $\mathrm{P}$ varying ninefold $\left(1.06-9.16 \mathrm{~g} / \mathrm{m}^{2}\right)$. N- and P-resorption efficiencies positively correlate with each other, as do senesced-leaf $\mathrm{N}$ and P. Consequently, species with high $\mathrm{N}$-resorption capacity have high P-resorption capacity. N-resorption efficiency is strongly negatively correlated with senesced-leaf $\mathrm{N}$ concentration, and P-resorption efficiency is strongly negatively correlated with senesced-leaf $\mathrm{P}$ concentration. Thus, species with high resorption efficiency capacity are also more proficient (Table 2).

$\mathrm{N}: \mathrm{P}$ resorption efficiency ratio is only significantly correlated with senesced-leaf $\mathrm{P}$ concentration. Senesced-leaf N:P ratio does not correlate with any resorption metric (Table 2).

N-resorption efficiency displays significant differences by growth form, with basal rosette perennials recovering more $\mathrm{N}$ relative to their nutrient pool than either annuals or erect perennials $(p=0.016)$. We find no significant differences by growth form for senesced-leaf $\mathrm{N}$ concentration, although basal rosette perennials have substantially lower green-leaf $\mathrm{N}$ concentration (Mason and Donovan 2015b). Thus, basal rosettes resorb more $\mathrm{N}$ than erect perennials and annuals relative to their nutrient pool, but have equivalent resorption proficiency.

Correlations between nutrient resorption and leaf economic traits

We defined resource-conservative leaf economic strategy by lower values of first principal components axis of LES traits (photosynthetic rate, respiration rate, leaf lifespan, LMA, and green-leaf $\mathrm{N}$ and $\mathrm{P}$ concentrations) from Mason and Donovan (2015b). This axis explains $56.2 \%$ of variation in area-based LES traits and $51.5 \%$ of variation in mass-based LES traits and 
Table $3 \mathrm{r}^{2}$ of evolutionary trait-environment correlations on population-weighted species means for (a) mass- and (b) areanormalized data

\begin{tabular}{|c|c|c|c|c|c|c|c|c|c|}
\hline 3 (a) & $\mathrm{P}_{\mathrm{eff}}$ & $\mathrm{P}_{\mathrm{SL}}$ & $\mathrm{N}_{\mathrm{eff}}$ & $\mathrm{N}_{\mathrm{SL}}$ & $N_{\text {eff }}: P_{\text {eff }}$ & $\mathrm{N}_{\mathrm{SL}}: \mathrm{P}_{\mathrm{SL}}$ & Flavonoid & Phenol & LSR \\
\hline Latitude & - & - & - & $(+) 0.17$ & - & - & - & - & - \\
\hline Longitude & - & - & - & (-) 0.23 & - & - & - & - & - \\
\hline Altitude & - & - & - & $(+) 0.16$ & - & - & - & - & - \\
\hline MAT & - & - & - & (-) 0.14 & - & - & - & - & - \\
\hline MDR & - & - & - & - & - & - & - & - & - \\
\hline ISO & - & - & - & - & - & - & - & - & - \\
\hline TS & - & - & - & - & - & - & - & - & - \\
\hline MTM $_{\text {warm }}$ & - & - & - & - & - & - & - & - & - \\
\hline MTM $_{\text {cold }}$ & - & - & - & - & - & - & - & - & - \\
\hline TAR & - & - & - & - & - & - & - & - & - \\
\hline $\mathrm{MTQ}_{\text {wet }}$ & - & - & - & - & - & - & $(-) 0.18$ & - & - \\
\hline $\mathrm{MTQ}_{\text {dry }}$ & - & - & - & - & - & - & - & - & - \\
\hline $\mathrm{MTQ}_{\text {warm }}$ & - & - & - & - & - & - & - & - & - \\
\hline $\mathrm{MTQ}_{\text {cold }}$ & - & - & - & - & - & - & - & - & - \\
\hline AP & - & - & - & $(-) 0.17$ & - & - & - & - & - \\
\hline $\mathrm{PM}_{\text {wet }}$ & - & - & - & $(-) 0.15$ & - & - & - & - & - \\
\hline $\mathrm{PM}_{\text {dry }}$ & - & - & - & $(-) 0.19$ & - & - & - & - & - \\
\hline PS & - & - & - & - & - & - & - & - & - \\
\hline$P_{\text {wet }}$ & - & - & - & $(-) 0.16$ & - & - & - & - & - \\
\hline$P_{\text {dry }}$ & - & - & - & $(-) 0.17$ & - & - & - & - & - \\
\hline$P Q_{\text {warm }}$ & - & - & - & - & - & - & - & - & - \\
\hline $\mathrm{PQ}_{\text {cold }}$ & - & - & - & $(-) 0.16$ & - & - & - & - & - \\
\hline PET & - & - & - & - & - & - & - & - & - \\
\hline ARI & - & - & - & - & - & - & - & - & - \\
\hline $\mathrm{N}_{\mathrm{GL}}: \mathrm{P}_{\mathrm{GL}}$ & - & - & - & - & - & - & - & - & - \\
\hline $\mathrm{C}_{\mathrm{GL}}: \mathrm{N}_{\mathrm{GL}}$ & - & - & - & - & - & - & - & - & - \\
\hline Soil N & - & - & - & - & - & - & $(+) 0.30$ & $(+) 0.32$ & - \\
\hline Soil C & - & - & - & - & - & - & - & $(+) 0.15$ & - \\
\hline Soil C:N & - & - & - & - & - & - & - & - & - \\
\hline Soil N:P & - & - & - & - & - & - & - & - & - \\
\hline Soil OM & - & - & - & - & - & - & $(+) 0.34$ & $(+) 0.42$ & - \\
\hline Soil P & - & - & - & - & - & - & - & - & - \\
\hline Soil K & - & - & - & - & - & - & - & - & - \\
\hline Soil Mg & - & - & - & - & - & $(-) 0.19$ & - & - & - \\
\hline Soil Ca & - & - & - & - & - & - & - & - & - \\
\hline Soil pH & - & $(+) 0.14$ & - & - & - & - & - & - & - \\
\hline Soil CEC & - & - & - & - & - & - & - & - & - \\
\hline $\mathrm{OM}, \mathrm{N}, \mathrm{C}$ & - & - & - & - & - & - & $(+) 0.25$ & $(+) 0.31$ & - \\
\hline $\mathrm{P}, \mathrm{K}, \mathrm{Mg}$ & - & - & - & - & - & - & - & - & - \\
\hline $\mathrm{Ca}, \mathrm{pH}, \mathrm{CEC}$ & - & - & - & - & - & - & - & - & - \\
\hline 3 (b) & $\mathrm{P}_{\text {eff }}$ & $\mathrm{P}_{\mathrm{SL}}$ & $\mathrm{N}_{\text {eff }}$ & $\mathrm{N}_{\mathrm{SL}}$ & $\mathrm{N}_{\mathrm{eff}}: \mathrm{P}_{\mathrm{eff}}$ & $\mathrm{N}_{\mathrm{SL}}: \mathrm{P}_{\mathrm{SL}}$ & Flavonoid & Phenol & LSR \\
\hline Latitude & - & - & - & - & - & - & - & - & - \\
\hline Longitude & - & - & - & $(-) 0.27$ & - & - & - & - & - \\
\hline
\end{tabular}


Table 3 continued

\begin{tabular}{|c|c|c|c|c|c|c|c|c|c|}
\hline 3 (b) & $P_{\text {eff }}$ & $\mathrm{P}_{\mathrm{SL}}$ & $\mathrm{N}_{\mathrm{eff}}$ & $\mathrm{N}_{\mathrm{SL}}$ & $\mathrm{N}_{\mathrm{eff}}: \mathrm{P}_{\mathrm{eff}}$ & $\mathrm{N}_{\mathrm{SL}}: \mathrm{P}_{\mathrm{SL}}$ & Flavonoid & Phenol & LSR \\
\hline Altitude & - & - & - & - & - & - & - & - & - \\
\hline MAT & - & - & - & - & - & - & - & - & - \\
\hline MDR & - & - & - & - & - & - & - & - & - \\
\hline ISO & - & - & - & - & - & - & - & - & - \\
\hline TS & - & - & - & - & - & - & - & - & - \\
\hline MTM $_{\text {warm }}$ & - & - & - & - & - & - & - & - & - \\
\hline MTM $_{\text {cold }}$ & - & - & - & - & - & - & - & - & - \\
\hline TAR & - & - & - & - & - & - & - & - & - \\
\hline $\mathrm{MTQ}_{\text {wet }}$ & - & - & - & - & - & - & $(-) 0.16$ & - & - \\
\hline $\mathrm{MTQ}_{\mathrm{dry}}$ & - & - & - & - & - & - & - & - & - \\
\hline $\mathrm{MTQ}_{\text {warm }}$ & - & - & - & - & - & - & - & - & - \\
\hline $\mathrm{MTQ}_{\text {cold }}$ & - & - & - & - & - & - & - & - & - \\
\hline $\mathrm{AP}$ & - & - & - & (-) 0.14 & - & - & - & - & - \\
\hline $\mathrm{PM}_{\text {wet }}$ & - & - & - & - & - & - & - & - & - \\
\hline $\mathrm{PM}_{\mathrm{dry}}$ & - & - & - & $(-) 0.15$ & - & - & - & - & - \\
\hline PS & - & - & - & - & - & - & - & - & - \\
\hline $\mathrm{PQ}_{\text {wet }}$ & - & - & - & - & - & - & - & - & - \\
\hline$P Q_{\text {dry }}$ & - & - & - & - & - & - & - & - & - \\
\hline$P Q_{\text {warm }}$ & - & - & - & - & - & - & - & - & - \\
\hline $\mathrm{PQ}_{\text {cold }}$ & - & - & - & - & - & - & - & - & - \\
\hline PET & - & - & - & - & - & - & - & - & - \\
\hline ARI & - & - & - & (-) 0.16 & - & - & - & - & - \\
\hline $\mathrm{N}_{\mathrm{GL}}: \mathrm{P}_{\mathrm{GL}}$ & - & - & - & - & - & - & - & - & - \\
\hline $\mathrm{C}_{\mathrm{GL}}: \mathrm{N}_{\mathrm{GL}}$ & - & - & - & - & - & - & - & - & - \\
\hline Soil N & - & - & - & - & - & - & $(+) 0.22$ & $(+) 0.26$ & - \\
\hline Soil C & - & - & - & - & - & - & - & - & - \\
\hline Soil C:N & - & - & - & - & - & - & - & - & - \\
\hline Soil N:P & - & - & - & - & - & - & - & - & - \\
\hline Soil OM & - & - & - & - & - & - & $(+) 0.25$ & $(+) 0.32$ & - \\
\hline Soil P & - & - & - & - & - & - & - & - & - \\
\hline Soil K & - & - & - & - & - & - & - & - & - \\
\hline Soil Mg & - & - & - & - & - & $(-) 0.19$ & - & - & - \\
\hline Soil Ca & - & - & - & - & - & - & - & - & - \\
\hline Soil pH & - & - & - & - & - & - & - & - & - \\
\hline Soil CEC & - & - & - & - & - & - & - & - & - \\
\hline $\mathrm{OM}, \mathrm{N}, \mathrm{C}$ & - & - & - & - & - & - & $(+) 0.17$ & $(+) 0.24$ & - \\
\hline $\mathrm{P}, \mathrm{K}, \mathrm{Mg}$ & - & - & - & - & - & - & - & - & - \\
\hline $\mathrm{Ca}, \mathrm{pH}, \mathrm{CEC}$ & - & - & - & - & - & - & - & - & - \\
\hline
\end{tabular}

All values shown are significant at $\mathrm{p} \leq 0.05$. Bold correlations are significant under FDR multiple comparisons. MAT mean annual temperature; $M D R$ mean diurnal range, ISO isothermality (diurnal range/annual range of temperature), $T S$ temperature seasonality, $M T M_{\text {warm }}$ max temperature of warmest month, $M T M_{\text {cold }}$ min temperature of coldest month, $T A R$ temperature annual range, $M T Q_{\text {wet }}$ mean temperature of wettest quarter, $M T Q_{d r y}$ : mean temperature of driest quarter, $M T Q_{\text {warm }}$ mean temperature of warmest quarter, $M T Q_{\text {cold }}$ mean temp of coldest quarter, $A P$ annual precipitation, $P M_{w e t}$ precipitation of wettest month, $P M_{d r y}$ precipitation of driest month, PS: precipitation seasonality, $P Q_{\text {wet }}$ precipitation of wettest quarter, $P Q_{d r y}$ precipitation of driest quarter, $P Q_{\text {warm }}$ precipitation of warmest quarter, $P Q_{\text {cold }}$ precipitation of coldest quarter, $A R I$ aridity index, $O M$ organic matter, $C E C$ cation exchange capacity, soil $\mathrm{PC} 1$ of $\mathrm{OM}, \mathrm{N}$, and $\mathrm{C}$; Soil PC1 of $\mathrm{P}, \mathrm{K}$, and $\mathrm{Mg}$; Soil PC1 of $\mathrm{Ca}, \mathrm{pH}$, and CEC 
(Mason and Donovan 2015b). Under area normalization, species with lower senesced-leaf nutrient concentration have a resource-conservative leaf economic strategy. Thus, resource-conservative species (i.e., species with lower photosynthetic and respiration rates and longer leaf lifespan) are more proficient. Senesced-leaf N (i.e., N proficiency) is evolutionarily correlated with every leaf economic trait except leaf lifespan and nighttime respiration rate (Table 2b). All significant correlations are in the direction predicted by the hypothesis that more complete nutrient resorption proficiency is a resource conservative trait except for the correlation with LMA. Senesced-leaf P (P proficiency) only correlates with two LES traits (respiration rate and green-leaf $\mathrm{P}$ concentration; Table 2b).

Mass normalization does not show an association between senesced-leaf nutrient concentration and the principal components based assessment of LES as a whole. However, senesced-leaf $\mathrm{N}$ correlates positively with leaf lifespan, and senesced-leaf $\mathrm{P}$ correlates positively with nighttime respiration (Table $2 \mathrm{a}$ ). Leaf senescence rate correlates positively with leaf lifespan, but not with any measure of nutrient resorption (Table 2). N:P resorption-efficiency ratios correlate positively with nighttime respiration rate.

Correlated trait evolution of chemical leaf defenses, but not leaf vascular capacity, with resorption proficiency

Leaf phenolic content correlates negatively with senesced-leaf $\mathrm{N}$ and $\mathrm{P}$ concentrations regardless of normalization (Table 2). Tannin activity is negatively correlated with senesced-leaf $\mathrm{N}$ concentration under mass normalization (Table 2a). Thus, species with more leaf total phenolic compounds are more $\mathrm{N}$ and $\mathrm{P}$ proficient, and species with higher tannin content are more $\mathrm{N}$ proficient. Leaf minor vein density does not exhibit correlated evolution with nutrient resorption (Table 2).

Correlations between nutrient resorption and soil fertility and precipitation

In general, native habitat soil fertility does not correlate with nutrient resorption. N- and P-resorption efficiencies and area-based normalizations of $\mathrm{N}$ - and P-resorption proficiencies (senesced-leaf $\mathrm{N}$ and $\mathrm{P}$ ) do not correlate with any native habitat soil fertility measurement (Table 3). Mass-based senesced-leaf $\mathrm{P}$ concentration only correlates with soil $\mathrm{pH}$, although this correlation loses significance under FDR correction; mass-based senesced-leaf $\mathrm{N}$ concentration does not correlate with any measure of fertility (Table 3 ).

In general, senesced-leaf nutrient concentration decreases in warmer habitats with more precipitation (Table 3). Under mass-based normalization, senesced-leaf $\mathrm{N}$ decreases as mean annual temperature increases (Table 3a). Multiple precipitation measures also correlate negatively with mass-based senescedleaf $\mathrm{N}$ concentration, with species evolving more complete $\mathrm{N}$ proficiency in habitats with more precipitation. Senesced-leaf N concentration correlates negatively with annual precipitation and precipitation of the driest month under area normalization, though these correlations lose significance under FDR correction. Senesced-leaf $\mathrm{P}$ concentration and $\mathrm{N}-$ and P-resorption efficiency do not correlate with climate (Table 3).

\section{Discussion}

Evolution of nutrient resorption as a resourceconservative trait

Given the importance of foliar nutrient resorption to plant nutrient economy, determining whether nutrient resorption capacity corresponds with other trait syndromes is key to understanding plant adaptive strategy. Under area normalization, we find more complete resorption proficiency in resource-conservative Helianthus species, as defined by the first principal components axis of LES traits (photosynthetic rate, respiration rate, leaf lifespan, LMA, and green-leaf $\mathrm{N}$ and $\mathrm{P}$ concentrations) from Mason and Donovan (2015b). This is consistent with the hypothesis that resource-conservative species resorb more foliar nutrients than resource-acquisitive species (Wood et al. 2011; Wright and Westoby 2003; Zhang et al. 2015). However, under mass normalization, more complete resorption proficiency is not found in more resource-conservative Helianthus species, likely due to some of the unique aspects of bivariate LES trait relationships for Helianthus.

The bivariate relationships of resorption proficiency with individual leaf economic traits in 
Helianthus are not always as might be expected based on broad cross-species patterns of LES (Diaz et al. 2004; Wright et al. 2004; but see Edwards et al. 2014 and Funk and Cornwell 2013). For example, higherLMA leaves display less complete area-based resorption. This is probably because for this study system, LMA positively correlates with area-based photosynthetic rate $\left(A_{\text {area }}\right)$ and not leaf lifespan (Mason and Donovan 2015b). At smaller phylogenetic scales such as within deciduous Viburnum, higher LMA occurs via thicker layers of photosynthetically active mesophyll cells (Edwards et al. 2014). More mesophyll cells increase LMA and $A_{\text {area }}$ simultaneously because leaf area remains constant, while the photosynthetically active cells increase. Meanwhile, LMA remains negatively correlated with mass-based photosynthetic rate because increased mass from these cells dilutes mass-based photosynthetic gains (Edwards et al. 2014). It is not known if a similar association exists between mesophyll cell layers and LMA in Helianthus species; however, the positive relationship between LMA and $A_{\text {area }}$ suggests it may. Since $A_{\text {area }}$ and LMA may be mechanistically related, a similarly directional relationship between nutrient resorption and both these LES traits may be unavoidable.

Additionally, leaf lifespan does not correlate with senesced-leaf nutrient concentration in this herbaceous study system, where leaf lifespan is driven by growing season length and may not be influenced by selective pressure on mean retention time (Mason and Donovan 2015b; Mason et al. 2017a). We further find evolution of extended leaf senescence does not account for variation in nutrient resorption, nor the relationship between leaf economic strategy and nutrient resorption capacity, in Helianthus. Instead, leaf economic strategy and nutrient resorption appear to be governed by whole-plant processes ultimately driven by growing season and relative growth rate.

Thus, although $\mathrm{N}$ and $\mathrm{P}$ resorptions are strongly correlated with each other and some traits associated with resource-use strategy, they are still labile enough to evolve independent associations with other traits. These disparate relationships additionally support the emerging understanding that leaf economics strategy is a constellation of interacting traits rather than a tight linear spectrum (Mason and Donovan 2015b; Poorter et al. 2014).
Nutrient resorption correlates differently with other leaf traits than predicted by the literature

Researchers have hypothesized that leaf phenolics inhibit resorption by precipitating proteins (Aerts 1997; Chapin and Kedrowski 1983). In contrast, our data show that neither tannin activity (as measured by protein-precipitation capacity) nor total phenolics (as measured by Folin-Ciocalteau assay) constrain resorption. Indeed, higher phenolic content and tannin activity evolves across Helianthus as mass-based $\mathrm{N}$-resorption proficiency becomes more complete. However, the absolute value of tannins in this herbaceous genus ranged from 0.005 to $2.516 \%$ tannic acid equivalents per leaf dry mass, which is much lower than levels reported in many woody species. This highlights the need to include herbaceous species in resorption studies because they often occupy different trait space than more heavily studied functional groups like trees. Studying herbaceous systems can provide demarcation for these hypotheses. For example, our data suggest that phenolics may not influence resorption at concentrations lower than $2.5 \%$. Our data support the hypothesis that highly proficient species evolve higher secondary metabolite concentrations to defend the $\mathrm{N}$ and $\mathrm{P}$ fated to be recycled from senescing leaves. High molecular weight tannins increase with mass-based $\mathrm{N}$ proficiency in Helianthus. Tannins also increase as resource conservatism increases (Mason et al. 2016). These relationships generally explain less than $25 \%$ of regression variation, indicating direct physiological dependencies are not driving these correlations (Poorter et al. 2014). Overall, resorption, secondary metabolites, and resource economic strategy represent different axes of variation interacting based on more than just their shared carbon and nutrient economics.

Our data do not show any correlated evolution between leaf vein density and nutrient resorption. Rather, increased leaf vein density associates with a resource-acquisitive strategy in Helianthus (Mason and Donovan 2015b), suggesting high nutrient resorption is possible with relatively low leaf vein density. In their 2015 dipterocarp study, Zhang et al. found leaf vein density was strongly positively associated with nutrient resorption. While we cannot explain why our study system shows a different pattern, lack of evolutionary correlation between these two traits is not surprising since processes related to water supply 
and photosynthate export mobilize orders of magnitude more material than processes involved in nutrient resorption.

Greater nutrient resorption capacity is beneficial in a variety of habitats

Our data show Helianthus species adapting to lowerfertility environments do not evolve higher resorption capacity. This finding differs from studies within global- and community-level datasets that show higher nutrient resorption capacity among species in lower-fertility environments (Aerts 1996; Killingbeck 1996; Kobe et al. 2005; Wright and Westoby 2003; Yuan and Chen 2009). These broad datasets are useful for determining nutrient-cycling dynamics within and across ecosystems, but cannot produce compelling evidence of evolutionary adaptation or underlying genetic and physiological linkages because they encompass many different variation sources. Our study prevents or accounts for environmental, phylogenetic, and ontogenetic variation sources. We acknowledge our single time-point soil sampling may not be a reliable proxy for whole-season fertility due to flushes and leaching that keep soil nutrients in constant flux. Still, given the wide variation in habitat occupancy across the genus (e.g., deserts to wetlands), the overall pattern obtained via our soil sampling is most likely sufficient for summarizing patterns of soil composition in habitats occupied by the diverse Helianthus genus.

Green-leaf $\mathrm{N}: \mathrm{P}$ and $\mathrm{C}: \mathrm{N}$ ratios, which are often used as proxies for nutrient limitation (Han et al. 2013), did not correspond to any resorption metric, including N:P resorption efficiency and proficiency ratios. This finding is not entirely unexpected, since $\mathrm{N}: \mathrm{P}$ ratio is a poor proxy for nutrient limitation in other species (Drenovsky and Richards 2004), and poor nutrient resorption capacity actually reinforces plantlevel nutrient limitation in desert shrubs (Drenovsky and Richards 2006). In contrast to soil fertility, climate predicts $\mathrm{N}$-resorption proficiency. In general, $\mathrm{N}$ proficiency follows the same pattern as the LES, with resource-conservative, $\mathrm{N}$-proficient species occupying warmer and/or wetter habitats than their resourceacquisitive, less proficient congenerics.

In conclusion, our results show that nutrient resorption falls within the general framework of the LES in this phenotypically diverse herbaceous genus.
Fast-growing, resource-acquisitive, annual Helianthus species live in lower-precipitation habitats and exhibit lower resorption capacity. Meanwhile, slow-growing, resource-conservative basal rosette perennial species in warm and rainy habitats have evolved higher resorption capacity. Furthermore, the role of leaf phenolics in protecting green-leaf resources appears compatible with the role of resorption in conserving leaf resources during senescence. Our results suggest nutrient resorption evolution may be tied to resource economics strategy more than native environment, with resource-conservative, proficient species capable of thriving in a variety of habitats.

Acknowledgements The authors wish to thank M. Boyd and $\mathrm{K}$. Tarner for assistance in plant growth and greenhouse maintenance, C. Uzoigwe for assistance collecting dead-leaf nutrient concentrations, S. McGaughey for assistance assessing leaf lifespan data, and E. Goolsby for assistance with implementing phylogenetic comparative models. We additionally wish to thank the Donovan lab group for helpful comments on the manuscript. This research was funded by National Science Foundation Grants 1122842 and 1444522 to L.A.D.

\section{Compliance with ethical standards}

Conflict of interest The authors declare that they have no conflict of interest.

Open Access This article is distributed under the terms of the Creative Commons Attribution 4.0 International License (http:// creativecommons.org/licenses/by/4.0/), which permits unrestricted use, distribution, and reproduction in any medium, provided you give appropriate credit to the original author(s) and the source, provide a link to the Creative Commons license, and indicate if changes were made.

\section{References}

Aerts R (1996) Nitrogen resorption from senescing leaves of perennials: are there general patterns? J Ecol 84:597-608. https://doi.org/10.2307/2261481

Aerts R (1997) Nitrogen partitioning between resorption and decomposition pathways: a trade-off between nitrogen use efficiency and litter decomposibility? Oikos 80:603-606. https://doi.org/10.2307/3546636

Aerts R, Chapin FS (2000) The mineral nutrition of wild plants revisited: a re-evaluation of processes and patterns. Adv Ecol Res 30:1-67

Benjamini Y, Hochberg Y (1995) Controlling the false discovery rate-a practical and powerful approach to multiple testing. J R Stat Soc B 57:289-300

Blomberg SP, Garland T, Ives AR (2003) Testing for phylogenetic signal in comparative data: behavioral traits are 
more labile. Evolution 57:717-745. https://doi.org/10. 1111/j.0014-3820.2003.tb00285.x

Bowsher AW, Miller BJ, Donovan LA (2016) Evolutionary divergences in root system morphology, allocation, and nitrogen uptake in species from high-versus low-fertility soils. Funct Plant Biol 43:129-140. https://doi.org/10. 1071/fp 15162

Brant AN, Chen HYH (2015) Patterns and mechanisms of Nutrient resorption in plants. CRC Crit Rev Plant Sci 34:471-486. https://doi.org/10.1080/07352689.2015. 1078611

Bruggerman J, Heringa J, Brandt BW (2009) PhyloPars: estimation of missing parameter values using phylogeny. Nucleic Acids Res 37:W179-W184. https://doi.org/10. 1093/nar/gkp370

Chapin FS (1980) The mineral nutrition of wild plants. Annu Rev Ecol Syst 11:233-260

Chapin FS (1989) The cost of tundra plant structures-evaluation of concepts and currencies. Am Nat 133:1-19

Chapin FS, Kedrowski RA (1983) Seasonal-changes in nitrogen and phosphorus fractions and autumn retranslocation in evergreen and deciduous taiga trees. Ecology 64:276-391. https://doi.org/10.2307/1937083

de Villemereuil P, Gaggiotti OE, Mouterde M, Till-Bottraud I (2016) Common garden experiments in the genomic era: new perspectives and opportunities. Heredity 116:249-254. https://doi.org/10.1038/hdy.2015.93

Diaz S, Hodgson JG et al (2004) The plant traits that drive ecosystems: evidence from three continents. J Veg Sci 15:295-304. https://doi.org/10.1111/j.1654-1103.2004. tb02266.x

Distel RA, Moretto AS, Didone NG (2003) Nutrient resorption from senescing leaves in two Stipa species native to central Argentina. Austral Ecol 28:210-215. https://doi.org/10. 1046/j.1442-9993.2003.01269.x

Donovan LA, Mason CM, Bowsher AW, Goolsby EW, Ishibashi CDA (2014) Ecological and evolutionary lability of plant traits affecting carbon and nutrient cycling. J Ecol 102:302-314

Drenovsky RE, Richards JH (2004) Critical N: P values: predicting nutrient deficiencies in desert shrublands. Plant Soil 259:59-69. https://doi.org/10.1023/B:PLSO.0000020945. 09809.3d

Drenovsky RE, Richards JH (2006) Low leaf N and P resorption contributes to nutrient limitation in two desert shrubs. Plant Ecol 183:305-314. https://doi.org/10.1007/s11258-0059041-z

Eckstein RL, Karlsson PS, Weih M (1999) Leaf life span and nutrient resorption as determinants of plant nutrient conservation in temperate-arctic regions. N Phytol 143:177-189. https://doi.org/10.1046/j.1469-8137.1999. 00429.x

Edwards EJ, Chatelet DS, Sack L, Donoghue MJ (2014) Leaf life span and the leaf economic spectrum in the context of whole plant architecture. J Ecol 102:328-336

Felsenstein J (1985) Phylogenies and the comparative method. Am Nat 125:1-15. https://doi.org/10.1086/284325

Freschet GT, Cornelissen JHC, van Logtestijn RSP, Aerts R (2010) Substantial nutrient resorption from leaves, stems and roots in a subarctic flora: what is the link with other resource economics traits? N Phytol 186:879-889. https:// doi.org/10.1111/j.1469-8137.2010.03228.x

Funk JL, Cornwell WK (2013) Leaf traits within communities: context may affect the mapping of traits to function. Ecology 94:1893-1897. https://doi.org/10.1890/12-1602.1

Goolsby EW (2016) Likelihood-based parameter estimation for high-dimensional phylogenetic comparative models: overcoming the limitations of "distance-based" methods. Syst Biol 65:852-870. https://doi.org/10.1093/sysbio/ syw051

Han W, Tang L, Chen Y, Fang J (2013) Relationship between the relative limitation and resorption efficiency of nitrogen vs phosphorus in woody plants. PLoS ONE. https://doi.org/ 10.1371/journal.pone. 0083366

Heiser CBJ, Smith DM, Clevenger SB, Martin WCJ (1969) The North American sunflowers: Helianthus. Memoirs Torrey Bot Club 22:1-218

Hijmans RJ, Cameron SE, Parra JL, Jones PG, Jarvis A (2005) Very high resolution interpolated climate surfaces for global land areas. Int J Climatol 25:1965-1978

Kandil FE, Grace MH, Seigler DS, Cheeseman JM (2004) Polyphenolics in Rhizophora mangle L. leaves and their changes during leaf development and senescence. TreesStruct Funct 18:518-528. https://doi.org/10.1007/s00468004-0337-8

Killingbeck KT (1996) Nutrients in senesced leaves: keys to the search for potential resorption and resorption proficiency. Ecology 77:1716-1727. https://doi.org/10.2307/2265777

Kobe RK, Lepczyk CA, Iyer M (2005) Resorption efficiency decreases with increasing green leaf nutrients in a global data set. Ecology 86:2780-2792. https://doi.org/10.1890/ 04-1830

Lloyd J, Bloomfield K, Domingues TF, Farquhar GD (2013) Photosynthetically relevant foliar traits correlating better on a mass vs an area basis: of ecophysiological relevance or just a case of mathematical imperatives and statistical quicksand? New Phytol 199:311-321. https://doi.org/10. 1111/nph.12281

Martins EP, Garland T (1991) Phylogenetic analyses of the correlated evolution of continuous characters - a simulation study. Evolution 45:534-557. https://doi.org/10.2307/ 2409910

Mason CM, Donovan LA (2015a) Does investment in leaf defenses drive changes in leaf economic strategy? A focus on whole-plant ontogeny. Oecologia 177:1053-1066. https://doi.org/10.1007/s00442-014-3177-2

Mason CM, Donovan LA (2015b) Evolution of the leaf economics spectrum in herbs: evidence from environmental divergences in leaf physiology across Helianthus (Asteraceae). Evolution 69:2705-2720. https://doi.org/10.1111/ evo. 12768

Mason CM, McGaughey SE, Donovan LA (2013) Ontogeny strongly and differentially alters leaf economic and other key traits in three diverse Helianthus species. J Exp Bot 64:4089-4099. https://doi.org/10.1093/jxb/ert249

Mason CM, Bowsher AW, Crowell BL, Celoy RM, Tsai C-J, Donovan LA (2016) Macroevolution of leaf defenses and secondary metabolites across the genus Helianthus. N Phytol 209:1720-1733. https://doi.org/10.1111/nph. 13749 
Mason CM, Goolsby EW, Davis KE, Bullock DV, Donovan LA (2017a) Importance of whole-plant biomass allocation and reproductive timing to habitat differentiation across the North American sunflowers. Ann Bot 119:1131-1142. https://doi.org/10.1093/aob/mcx002

Mason CM, Patel HS, Davis KE, Donovan LA (2017b) Beyond pollinators: evolution of floral architecture with environment across the wild sunflowers (Helianthus, Asteraceae). Plant Ecol Evol 150:139-150. https://doi.org/10.5091/ plecevo.2017.1321

Osnas JLD, Lichstein JW, Reich PB, Pacala SW (2013) Global leaf trait relationships: mass, area, and the leaf economics spectrum. Science 340:741-744. https://doi.org/10.1126/ science. 1231574

Pagel M (1999) Inferring the historical patterns of biological evolution. Nature 401:877-884. https://doi.org/10.1038/ 44766

Paradis E, Claude J, Strimmer K (2004) APE: analyses of phylogenetics and evolution in $\mathrm{R}$ language. Bioinformatics 20:289-290

Pearson K (1897) Mathematical contributions to the theory of evolution: on a form of spurious correlation which may arise when indices are used in the measurement of organs. P R Soc London 60:489-498

Pilote AJ, Donovan LA (2016) Evidence of correlated evolution and adaptive differentiation of stem and leaf functional traits in the herbaceous genus, Helianthus. Am J Bot 103:2096-2104. https://doi.org/10.3732/ajb.1600314

Pinheiro J, Bates D, DebRoy S, Sarkar D and R Core Team (2014) nlme: linear and nonlinear mixed effects models. R package version 3.1-118. Retrieved from http://cran.rproject.org/package $=$ nlme

Poorter H, Lambers H, Evans JR (2014) Trait correlation networks: a whole-plant perspective on the recently criticized leaf economic spectrum. N Phytol 201:378-382. https:// doi.org/10.1111/nph.12547

Reich PB (2014) The world-wide 'fast-slow' plant economics spectrum: a traits manifesto. J Ecol 102:275-301

Revell LJ (2012) phytools: an R package for phylogenetic comparative biology (and other things). Methods Ecol Evol 3:217-223. https://doi.org/10.1111/j.2041-210X.2011. 00169.x

Sack L, Scoffoni C et al (2012) Developmentally based scaling of leaf venation architecture explains global ecological patterns. Nat Commun. https://doi.org/10.1038/ ncomms 1835

Sack L, Scoffoni C, John GP, Poorter H, Mason CM, MendezAlonzo R, Donovan LA (2013) How do leaf veins influence the worldwide leaf economic spectrum? Review and synthesis. J Exp Bot 64:4053-4080. https://doi.org/10.1093/ jxb/ert316
Stephens JD, Rogers WL, Mason CM, Donovan LA, Malmberg RL (2015) Species tree estimation of diploid Helianthus (Asteraceae) using target enrichment. Am J Bot 102:910-920. https://doi.org/10.3732/ajb.1500031

Tu YK (2016) Testing the relation between percentage change and baseline value. Sci Rep-UK. https://doi.org/10.1038/ srep23247

van Heerwaarden LM, Toet S, Aerts R (2003) Current measures of nutrient resorption efficiency lead to a substantial underestimation of real resorption efficiency: facts and solutions. Oikos 101:664-669. https://doi.org/10.1034/j. 1600-0706.2003.12351.x

Varvel G, Peterson G, Anderson F (1976) A revised method for determining phosphate-phosphorus levels in sugar beet leaf petioles. J Sugarbeet Res 19:138-142. https://doi.org/10. 5274/jsbr.19.2.138

Webber JF, Mason CM (2016) Utility of the colorimetric folinciocalteu and aluminum complexation assays for quantifying secondary metabolite variation among wild sunflowers. Helia. https://doi.org/10.1515/helia-2016-0019

Westoby M, Reich PB, Wright IJ (2013) Understanding ecological variation across species: area-based vs mass-based expression of leaf traits. N Phytol 199:322-323. https://doi. org/10.1111/nph.12345

Wood TE, Lawrence D, Wells JA (2011) Inter-specific variation in foliar nutrients and resorption of nine canopy-tree species in a secondary neotropical rain forest. Biotropica 43:544-551. https://doi.org/10.1111/j.1744-7429.2010. 00740.x

Wright IJ, Westoby M (2003) Nutrient concentration, resorption and lifespan: leaf traits of Australian sclerophyll species. Funct Ecol 17:10-19. https://doi.org/10.1046/j.1365-2435. 2003.00694.x

Wright IJ, Reich PB et al (2004) The worldwide leaf economics spectrum. Nature 428:821-827. https://doi.org/10.1038/ nature 02403

Yuan ZY, Chen HYH (2009) Global trends in senesced-leaf nitrogen and phosphorus. Global Ecol Biogeogr 18:532-542. https://doi.org/10.1111/j.1466-8238.2009. 00474.x

Zhang J-L, Zhang S-B, Chen Y-J, Zhang Y-P, Poorter L (2015) Nutrient resorption is associated with leaf vein density and growth performance of dipterocarp tree species. J Ecol 103:541-549. https://doi.org/10.1111/1365-2745.12392

Zomer RJ, Trabucco A, Bossio DA, Verchot LV (2008) Climate change mitigation: a spatial analysis of global land suitability for clean development mechanism afforestation and reforestation. Agr Ecosyst Environ 126:67-80 